\title{
Gradhiva
}

Revue d'anthropologie et d'histoire des arts

\section{Brice Gérard, Histoire de l'ethnomusicologie en France (1929-1961)}

Paris, L'Harmattan, coll. « Histoire des sciences humaines », 2014

\section{Marie-Barbara Le Gonidec}

\section{OpenEdition}

\section{Journals}

Édition électronique

URL : http://journals.openedition.org/gradhiva/3205

DOI : 10.4000/gradhiva.3205

ISSN : 1760-849X

\section{Éditeur}

Musée du quai Branly Jacques Chirac

Édition imprimée

Date de publication : 25 mai 2016

Pagination : 228-231

ISBN : 978-2-35744-093-7

ISSN : 0764-8928

\section{Référence électronique}

Marie-Barbara Le Gonidec, « Brice Gérard, Histoire de l'ethnomusicologie en France (1929-1967) »,

Gradhiva [En ligne], 23 | 2016, mis en ligne le 25 mai 2016, consulté le 23 septembre 2020. URL : http:// journals.openedition.org/gradhiva/3205; DOI : https://doi.org/10.4000/gradhiva.3205

Ce document a été généré automatiquement le 23 septembre 2020.

(c) musée du quai Branly 


\section{Brice Gérard, Histoire de} l'ethnomusicologie en France (1929-1961)

Paris, L'Harmattan, coll. « Histoire des sciences humaines », 2014

Marie-Barbara Le Gonidec

\section{RÉFÉRENCE}

Brice Gérard, Histoire de l'ethnomusicologie en France (1929-1961). Paris, L'Harmattan, coll. « Histoire des sciences humaines », 2014, 363 p. 


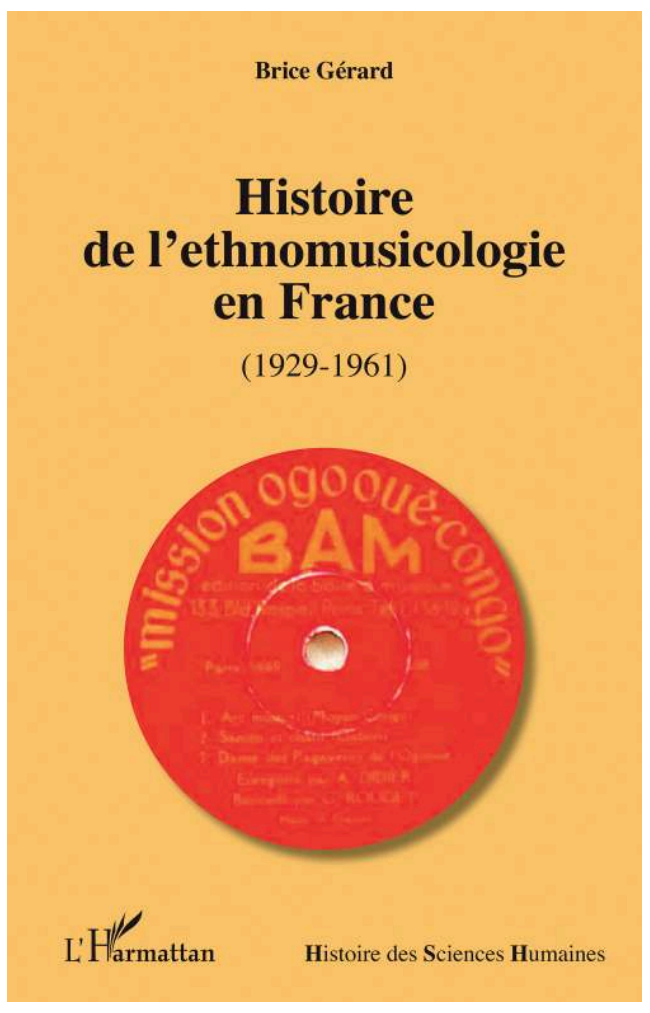

1 Alors que les deux grandes institutions qui l'ont fait naître et s'épanouir ont été fermées (de 2009 à 2015 pour le musée de l'Homme et depuis 2013 pour celui des Arts et Traditions populaires), alors que les données d'enquêtes et les archives produites par les pionniers du domaine (André Schaeffner au musée de l'Homme, Claudie MarcelDubois aux Arts et Traditions populaires) ont rejoint le Muséum et l'université de Nanterre pour les premières, les Archives nationales pour les secondes, le temps est venu de se pencher sur l'histoire de l'ethnomusicologie en France. Si quelques chercheurs ont proposé un retour réflexif sur leur propre discipline et l'évolution de ses problématiques, l'ouvrage de Brice Gérard s'appuie sur l'analyse détaillée de très nombreuses archives, une voie que les ethnomusicologues n'avaient encore jamais explorée. Le résultat offre une perspective nouvelle, d'autant que l'auteur a souhaité parler au singulier de l'histoire d'une discipline née sous le sceau d'une forte dichotomie entre les deux musées héritiers du musée d'Ethnographie du Trocadéro créés en 1937 -, qui n'a cessé de se perpétuer depuis au point de laisser croire à l'existence de deux branches structurellement différentes de l'ethnomusicologie : l'une traitant de l'exotique, l'autre du populaire.

2 L'histoire commence huit ans avant cette scission avec le recrutement, en 1929, du musicologue et critique musical André Schaeffner (1895-1980) par Georges Henri Rivière. Ce dernier, futur directeur du musée national des Arts et Traditions populaires est, depuis juin 1928, l'assistant de Paul Rivet, qui a entrepris la rénovation du musée d'Ethnographie du Trocadéro. L'ouverture d'un nouveau service, celui d'organologie musicale regroupant les instruments du musée, constitue l'acte fondateur, au plan institutionnel, de la discipline. Trois décennies plus tard, la mise en place d'un enseignement régulier montre que celle-ci s'est consolidée. En 1961 en effet, à l'initiative de Claude Lévi-Strauss, Claudie Marcel-Dubois (1913-1989) devient responsable du premier séminaire d'ethnomusicologie générale donné à l'École pratique des hautes études. Ce domaine, branche de l'ethnologie pour certains, de la 
musicologie pour d'autres, en se dégageant de cette double tutelle achève ainsi de s'ériger en discipline autonome.

3 L'auteur a choisi de découper l'ouvrage en trois parties, chacune correspondant à une décennie : 1929-1939, 1940-1949 et 1950-1961. L'analyse des documents lui a également permis d'identifier quatre façons, pour les ethnomusicologues, d'accéder aux connaissances.

4 Tout d'abord, le mode graphique, relativement banal, va former l'activité initiale de lecture et d'écriture du spécialiste de musique et d'instruments, qui doit désormais appréhender son objet au plan anthropologique. Celui-ci doit aussi envisager la nouvelle muséo-« graphie » des collections organologiques. Ce mode est également lié à la prise de notes sur le terrain (celles de Schaeffner feront de lui un africaniste) et à la restitution livresque des connaissances. Le deuxième mode, qualifié d'organologique l'organologie étant la science des instruments -, constitue logiquement l'activité principale de Schaeffner à ses débuts. Les archives montrent que "l'orientation épistémologique de l'ethnomusicologie qui s'institutionnalisait [a été] largement redevable [...] à une réflexion sur [...] l'instrument de musique » (p. 26). Schaeffner publie en 1936 chez Payot le monumental ouvrage Origine de la musique. Introduction ethnologique à l'histoire de la musique instrumentale, qui reste un classique. Conscient de leur transversalité, il choisit d'exposer les collections comme témoins de l'universalité des pratiques sonores plutôt que comme représentantes des différentes cultures musicales.

5 Longtemps limitée à la science des instruments - qui ne sont pourtant qu'un outil de la musique et non la musique elle-même -, l'étude de l'altérité musicale va prendre son essor grâce au mode audiovisuel. L'appareil enregistreur, plus que la caméra qui sera utilisée bien plus tard de manière systématique, est véritablement à l'origine de cette science dont l'intangibilité du matériau d'étude principal empêchait toute objectivation. Il libère de la transcription solfégique qui, mal adaptée aux musiques pour lesquelles ce code graphique n'avait pas été prévu, limitait considérablement le champ d'investigation, et surtout il rend possible d'appréhender toute production sonore et pas seulement musicale. Schaeffner l'utilise dès sa première enquête pour la mission Dakar-Djibouti, dont il est membre d'octobre 1931 à février 1932. La musique ainsi fixée sur un support devient alors un objet de musée, au même titre que l'instrument de musique. Cet élargissement va justifier le changement terminologique du «Service d'organologie», rebaptisé «Département d'ethnologie musicale». C'est aussi l'existence de collections de disques exigeant, comme toute collection muséale, d'être gérées et enrichies qui motivera le recrutement de Marcel-Dubois pour seconder Schaeffner à la phonothèque.

6 La mission Dakar-Djibouti, pourvoyeuse des tout premiers enregistrements de terrain de l'ethnomusicologie française, puis celles que Schaeffner mènera dans l'immédiat après-guerre avec Denise Paulme, devenue son épouse en 1937, vont faire du musicologue un ethnologue. Dès 1931, sa méthodologie, comme d'ailleurs celle de Marcel-Dubois sur son premier terrain en 1939, soumise au protocole du questionnaire, du journal de route et/ou du carnet de terrain, permet d'aborder les deux derniers modes dégagés par Gérard: le mode dialogique, qui renvoie à l'échange verbal avec l'informateur, et le mode observant. Entretiens et observations étant consignés par écrit, ces deux modes relèvent du mode graphique déjà évoqué, mais pas uniquement : leur analyse rend compte des idées et du vécu de l'ethnographe. En comparant les archives 
écrites des terrains «initiatiques » de Schaeffner (mission Dakar-Djibouti 1931) et de Marcel-Dubois (mission Basse-Bretagne 1939) devenus, par le jeu des choix institutionnels, ethnomusicologue « des Lointains » pour le premier, « du Proche » pour la seconde, l'auteur montre que l'observation directe revêtait dans ces années 1930 une importance relative, plus grande en Afrique qu'en France. Les notes de Schaeffner prouvent qu'il s'attache à observer le phénomène musical sous ses différents aspects sociaux, là où celles de Marcel-Dubois témoignent d'un respect scrupuleux du questionnaire établi avant le départ. Gérard en conclut que, ne cherchant pas comme Schaeffner à s'adapter au terrain, elle choisit de s'en tenir à une approche de type expérimental. Cela s'en ressent aussi à travers le qualificatif qu'elle emploie pour désigner les informateurs, ces " paysans de vieille race » auprès desquels elle a collecté le matériau d'étude via le mode audiovisuel. Schaeffner, pourtant lui aussi soumis au formalisme du questionnaire protocolaire, et surtout au contexte colonial qui hiérarchise les rapports humains, choisit néanmoins une tout autre posture en jouant le rôle d'apprenti percussionniste. L'incorporation de rythmes dogons au point d'en rendre la pratique "naturelle à sa pensée et de cesser de considérer cette musique comme nègre » (p. 107) montre sa capacité à abolir « l'extériorité constitutive de la relation d'un ethnologue avec son informateur» (ibid.) que Marcel-Dubois a souhaité maintenir. Cela est aussi révélateur de la «contemporanéité caractéristique de l'entreprise ethnographique dans les contextes lointains » (p. 130) qui s'oppose à la recherche du passé dans le présent observée pour la mission dans la péninsule armoricaine, encore considérée comme le dernier bastion de l'ancienne civilisation gauloise. Cela montre enfin que l'ethnomusicologie de la France se place d'emblée comme l'étude d'un exotisme de l'intérieur, la démarche "archéologique » instituant une distance temporelle là où l'étrangeté culturelle fait défaut.

7 Couvrant la période de l'Occupation, qui se traduit par un inévitable ralentissement des activités, la deuxième partie de l'ouvrage analyse les répercussions du contexte politique et idéologique de la guerre et de l'immédiat après-guerre. L'impact a surtout été conséquent au musée national des Arts et Traditions populaires, où Marcel-Dubois a tacitement fait cause commune avec Rivière, ce dernier ayant opportunément adopté les idées maréchalistes valorisant la paysannerie et l'artisanat. Alors qu'un réseau de résistants voyait le jour au musée de l'Homme et que Rivet, son directeur, quittait le territoire national, le musée national des Arts et Traditions populaires a en effet été tenté par un retour au folklorisme dont les objets conventionnels d'étude étaient non seulement réhabilités mais aussi « érigés en causes nationales» (p. 141). Schaeffner, qui connaissait Rivière depuis 1925 et auquel, on l'a vu, il devait sa carrière institutionnelle, soutiendra ce dernier quand il se verra inquiété en octobre 1944. Opportunisme n'étant pas allégeance, le directeur du musée national des Arts et Traditions populaires est rétabli quelques mois plus tard dans ses fonctions, et on lui reprochera simplement d'avoir été plus soucieux de mener à bien son projet que de résister à l'occupant. Cela s'est traduit par le recrutement de jeunes architectes et artistes dans le cadre des chantiers intellectuels consacrés au mobilier traditionnel, à l'architecture rurale et à l'artisanat, un personnel recruté à bon compte certes, mais que l'action de Rivière a mis à l'abri du travail obligatoire. Cette période peut en tout cas être qualifiée d'encombrante puisqu'elle pousse Marcel-Dubois, dans différents écrits rétrospectifs, à faire de 1945 l'année de naissance de l'ethnomusicologie de la France alors que l'analyse des archives ne rend compte d'aucune rupture dans sa démarche de 
recherche. Au contraire, elle va continuer à s'intéresser à la figure du paysan comme synonyme exclusif de populaire.

8 Pendant ce temps, au musée de l'Homme, on assiste à la modification des projets jusqu'alors au cœur de l'ethnologie musicale, suivant parfois l'évolution normale d'une discipline à l'issue de ses premières expériences, d'autres fois de façon plus radicale. L'époque, qui n'est plus propice aux grandes missions collectives, connaît par exemple une "autonomisation relative du travail scientifique» (p. 157). Le principal changement est insufflé par Gilbert Rouget (né en 1916), devenu, en 1942, l'assistant de Schaeffner. Il va contribuer à l'émergence d'une nouvelle « conception de l'articulation entre écriture et ethnographie» (p. 223). Pour Schaeffner, l'écrit, tout en alimentant une réflexion plus générale sur différentes formes artistiques, était l'aboutissement de l'enquête ethnographique. Pour Rouget, l'enquête est, dès l'origine, "destinée à constituer le commentaire du matériau sonore collecté en mission » (p. 223). Il écrira plus tard que « l'enregistrement sonore est, sinon la seule, du moins la plus importante source d'information pour l'ethnomusicologie ${ }^{1}{ }^{\prime}$ (p. 288). Il succédera à Schaeffner en 1965 et aura à cœur de mettre en place une politique éditoriale d'enregistrements sonores qu'il érigera quasiment en principe puisque ceux-ci constitueront un véritable mode d'écriture disciplinaire, même si, précisons-le, il n'est pas tenu compte des publications discographiques dans l'évaluation des chercheurs par le CNRS. Le musée national des Arts et Traditions populaires tentera, en vain, de développer une politique similaire. Ainsi, cette période est principalement caractérisée par le contraste entre deux conceptions: celle de Schaeffner, qui s'inscrit dans la continuité des missions Griaule où l'observation musicographique vient nourrir l'observation ethnographique ; et celle de Rouget, telle qu'il la consigne dans ses notes dactylographiées au retour de la mission Ogooué-Congo (1946), dont l'analyse invite Gérard à parler de "prééminence épistémologique de l'enregistrement sonore» (p. 158). Ceci vaut également pour les chercheuses du musée national des Arts et Traditions populaires - Maguy Andral (1922-2004) ayant rejoint Marcel-Dubois en décembre 1945 - dont les missions et opérations de sauvegarde s'inscrivent dans le cadre d'une ethnomusicologie d'urgence visant à conserver la trace mémorielle de pratiques moribondes.

9 C'est durant la décennie suivante et sous l'influence des grands théoriciens angloaméricains dont les travaux contribuent à nourrir la réflexion des chercheurs français que se fixe le nom d'une discipline désormais consolidée. Cependant, le terme s'impose plus qu'il ne fait consensus. Schaeffner lui préfère celui d'" ethnologie musicale", Rouget écrit «ethno-musicologie», et Marcel-Dubois l'abandonne en 1964 pour rebaptiser son service " Département de musique ethnique ${ }^{2}$ ». Malgré ces divergences, les ethnomusicologues des deux institutions forment une sorte de " collectif structuré " (p. 227) impliqué dans la communauté internationale par le biais de colloques et par leur affiliation à des réseaux professionnels : un collectif dont l'objet d'étude commun est la musique ethnique, et qui se distingue, au-delà des dissensions qui ne manquent pas d'exister, par une communauté de points de vue dont le principal est, partant du principe du phénomène acoustique comme fait social sonore, celui d'un élargissement du champ d'investigation à l'ensemble des manifestations vocales ou instrumentales. Ainsi, Marcel-Dubois, en prenant soin de ne pas considérer la chanson comme « représentant[e] unique de notre musique populaire» (p. 283), souhaite «donner au fait musical paysan de France un plus juste poids » (ibid.). La volonté de rupture avec les folkloristes est donc bien marquée, et vient contrebalancer le « repli frileux sur le paysan» (Christian Bromberger ${ }^{3}$, cité p. 245) qui caractérise la démarche des Arts et 
Traditions populaires. Le projet vise à couvrir l'ensemble du territoire national afin d'établir une sorte d'inventaire général des pratiques sonores et musicales. Du côté du musée de l'Homme, on assiste inversement à une spécialisation de terrain, Rouget choisissant d'approfondir ses recherches au Dahomey (actuel Bénin), Schaeffner restant spécialiste des Dogons (Mali) et des Kissi (Haute-Guinée).

Cette période qui va de 1950 à 1961 est enfin celle d'un tournant essentiel pour l'anthropologie. Le courant structuraliste secoue profondément l'ensemble de la communauté des chercheurs en sciences humaines. Qu'en est-il de celle des ethnomusicologues? Gérard s'attache à montrer que celle-ci n'a pas eu besoin d'emprunter le modèle lévi-straussien, possédant déjà un cadre défini dès les années 1930 par Constantin Brăiloiu. Roumain d'origine, venu étudier la musique à Paris, celuici fait de nombreuses collectes dans son pays où il fonde en 1928 les archives de folklore de la Société des compositeurs roumains. Établi en Suisse mais nommé au CNRS en 1948, il a l'occasion de partager son expérience avec Schaeffner et Marcel-Dubois, qu'il influence plus particulièrement en tant que spécialiste des musiques paysannes dont il est le principal théoricien. Dans « Esquisse d'une méthode de folklore musical ${ }^{4}$ » (1931), il propose la transcription d'un bocet (plainte funéraire de Transylvanie du Sud) sous une forme inédite : celle d'un tableau synoptique décomposant la structure de la pièce et tenant ainsi compte de l'ensemble des variations non pas comme variantes du modèle, mais comme système structurant les musiques de tradition orale. Il met donc au point une nouvelle méthodologie en musicologie, sa formation d'origine, permettant à sa branche ethnologique - dont il est vu aujourd'hui comme un des pères fondateurs de s'autonomiser.

11 Il convient pour finir de reparler de muséographie, étant donné l'importance des instruments dans le parcours professionnel de Schaeffner. Au musée de l'Homme, ceuxci sont exposés dans la salle des arts et techniques inaugurée en 1959 et partagée avec André Leroi-Gourhan. L'analyse de Gérard tend à montrer que Schaeffner a influencé le célèbre anthropologue, fondateur du Département de technologie comparée - l'autre service transversal de l'institution -, quant à la réalisation de cette salle. Celle-ci constitue la transposition muséographique d'une certaine conception de l'unité de l'homme (p. 238) fondée sur la théorie de Marcel Mauss: «Si les faits sociaux sont totaux, l'homme aussi est "total", faber et sapiens, à la fois producteur de biens matériels et créateur de langage symbolique " (Robert Cresswell ${ }^{5}$, cité p. 238). Il s'agit, en quelque sorte, d'une synthèse des travaux de ces deux grandes figures de l'anthropologie, développés dans leurs ouvrages respectifs, Origine des instruments de Schaeffner, L'Homme et la matière et Le Geste et la parole de Leroi-Gourhan. Cette muséographie, explique Gérard, donne autant à voir l'avancée en matière de savoir scientifique à cette époque que l'expression de la diversité des cultures humaines.

Cet ouvrage montre finalement que l'histoire de l'ethnomusicologie est bien celle d'une discipline, au sens où elle a fourni les repères méthodologiques indispensables à la réflexion sur l'altérité musicale qui constituait son principal objet d'étude. L'originalité de la méthode choisie par l'auteur, qui se pose ainsi en historien des sciences, a été de distinguer les différents "modes d'accès aux connaissances" des ethnomusicologues français entre 1929 et 1961, pour se pencher sur «l'évolution de leur articulation ». Si la démonstration repose essentiellement sur l'analyse des archives produites par les acteurs de l'institutionnalisation d'une discipline qu'ils contribuent à légitimer, celle-ci ne se réduit pas, précise Gérard à juste titre, à l'histoire des deux institutions majeures 
dont il est question au fil de l'ouvrage - l'accent étant mis sur le musée de l'Homme, un sous-titre aurait peut-être été bienvenu. Cette histoire doit en effet intégrer des savants considérés comme extérieurs ou marginaux tels que le folkloriste Patrice Coirault, spécialiste de la chanson, ou l'africaniste Herbert Pepper. Que serait par exemple l'histoire du folklore sans Arnold van Gennep ? Gérard démontre ainsi que l'historien ne doit pas reprendre à son compte les autodélimitations ou les délimitations rétrospectives du domaine, une entreprise sur ce plan réussie et qui apporte aux ethnomusicologues un regard nouveau sur leur discipline.

\section{NOTES}

1. Gilbert Rouget, « À propos de la forme dans les musiques de tradition orale », in Les Colloques de Wégimont (1954), Bruxelles, Elsevier, 1956 : 132-144.

2. Claudie Marcel-Dubois, Titres et travaux scientifiques, Paris, Impr. Circex, $1964: 4$, note 2. Elle travaille, écrit-elle encore, à la constitution du « corpus des musiques ethniques » («Papiers de l'équipe de recherche ethnomusicologique», mars 1972, documents dactylographiés, Paris, Archives nationales: 1). Ce qualificatif est étonnant pour désigner les musiques paysannes. Il pourrait témoigner de la nécessaire mise à distance propre à la démarche scientifique s'il ne traduisait pas, de fait, la crainte chez Claudie Marcel-Dubois, à cette époque encore, de voir son objet réduit au « folklore ».

3. Christian Bromberger, "L'ethnologie de la France, du Front populaire à la Libération : entre continuité et ruptures », in Jacqueline Christophe, Denis-Michel Boëll et Régis Meyran (éd.), Du folklore à l'ethnologie, Paris, Éditions de la maison des sciences de l'homme, 2009 : 1-10.

4. Constantin Brăiloiu, «Esquisse d'une méthode de folklore musical », Revue de musicologie 12(40), 1931 : 233-267.

5. Robert Cresswell, « Leroi-Gourhan André », in Pierre Bonte et Michel Izard (dir.), Dictionnaire de l'ethnologie et de l'anthropologie, Paris, PUF, 2007 [1991] : 414-416.

\section{AUTEURS}

\section{MARIE-BARBARA LE GONIDEC}

mb.legonidec@gmail.com 